\title{
Philosophiques
}

\section{Pierre Gravel, Pour une logique du sujet tragique. Sophocle, Montréal, Presses de l’Université de Montréal, 1980, 193 p.}

\section{Guy Bouchard}

Volume 8, numéro 2, octobre 1981

URI : https://id.erudit.org/iderudit/203178ar

DOI : https://doi.org/10.7202/203178ar

Aller au sommaire du numéro

Éditeur(s)

Société de philosophie du Québec

ISSN

0316-2923 (imprimé)

1492-1391 (numérique)

Découvrir la revue

Citer ce compte rendu

Bouchard, G. (1981). Compte rendu de [Pierre Gravel, Pour une logique du sujet tragique. Sophocle, Montréal, Presses de l'Université de Montréal, 1980, 193 p.] Philosophiques, 8(2), 358-360. https://doi.org/10.7202/203178ar d'utilisation que vous pouvez consulter en ligne.

https://apropos.erudit.org/fr/usagers/politique-dutilisation/ 
Pierre GRAVEL, Pour une logique du sujet tragique. Sopbocle, Montréal, Presses de l'Université de Montréal, 1980, 193 p.

par Guy Bouchard

Et si la tragédie [grecque (sophocléenne)] avait été la mise en scène de notre impossibilité? De «l'impossibilité pour ce que nous appelons un «sujet" de se constituer» (p.8)?

Tragédie et représentation. La tragédie est (forme particulière de) représentation. Mais la philosophie s'est elle-même voulue l'araignée régnant sur cette toile. En expulsant, platonicienne, le poète tragique, quitte à se prétendre elle-même poète; et tragique. «La tragédie, le discours tragique pourrait ainsi être le refoulé ou le refusé du discours philosophique, et, comme tel, éminemment significatif» (p. 13). Mais la tragédie est (forme particulière de) représentation. Et l'araignée, aujourd'hui, retisse cette toile: «penser la clôture de la représentation, dit Derrida, c'est penser le tragique». Comment la notion de représentation a-t-elle pu permettre ce déplacement? "La notion de représentation (...) s'entend d'abord d'une structure logique et conceptuelle, d'une relation dotée d'une efficace ontologique certaine, par quoi deux termes principaux sont produits, mis en scène et amenés, sinon tout de suite à paraître ( . . .), du moins à jouer et à travailler: le représenté, tout d'abord, de la représentation, c'est-à-dire cela qui est signifié, visé ou posé comme terme à la fois ultime et transcendant par le discours qui s'inscrit dans l'élément de la représentation ( . . . ); le représentant, ensuite, de la représentation, c'est-à-dire: celui pour qui et devant qui il y a une telle re-présentation, et qui ( . . .) n'est peut-être qu'un effet ou une fonction de cette structure de représentation", etc. (p. 14-16). Mais la représentation tragique? Elle a donné lieu a trois types principaux d'interprétation: l'un visant à en produire la structure (Aristote, Hölderlin); le second manifestant le registre de forces mises en jeu par cette structure (Aristote, Freud, Nietzsche); et le troisième tentant de révéler la signification ou le contenu d'une ou de plusieurs tragédies (Hegel, Brumoy). Cette dernière forme d'interprétation est la plus dangereuse, car elle risque de masquer la question que peut encore être pour nous la tragédie.

Problématiques. "Que la tragédie, et la tragédie grecque tout particulièrement, puisse toujours nous être question, c'est-à-dire nous être et se jouer de nous dans et par les termes mêmes que nous mettons en oeuvre pour l'interpéter, voilà certes ce qu'il faut tenter d'établir. Le soupçon qui nous guide: la tragédie serait un jeu, le type même d'interprétation que nous mettons en oeuvre à son propos serait inclus, mieux: serait partie prenante de la structure même du jeu qu'elle implique et déploie» (p. 47). Ainsi, pour déterminer les personnages de la tragédie, nous utilisons des termes, concepts et critères qui n'avaient par cours dans la langue grecque contemporaine des tragiques. Aristote l'a bien montré: l'action est l'âme de la tragédie, les «caractères» sont secondaires et même facultatifs; or Racine, "traduisant" Aristote, fait de 
l'action la manifestation du caractère, du "sujet»: il construit un lieu d'évidence qui est censé faire sens pour nous, mais à partir duquel l'analyse d'Aristote ne peut plus faire sens. Ainsi encore, pour déterminer les rapports de l'humain et du divin dans la tragédie, il est fait usage «de schémas de pensée dont le moins qu'on puisse dire est qu'ils répètent, et la plupart du temps dans une insouciance navrante, la scission ou la coupure originelle qui est le fonds du judéo-christianisme» (p.65). Or le désir qui préside à l'interprétation de la tragédie procède d'un désir de savoir qui se constitue, chez Platon par exemple, dans le refus et la relève du tragique, et qui se constitue dans «l'assignation à résidence de l'être de l'homme» (p. 70) comme disposant sinon déjà d'une nature, du moins de propriétés et d'attributs essentiels. Or «il se produirait entre autres choses ceci chez Sophocle qu'il serait impossible - la tragédie étant la monstration de cette impossibilité - d'assigner à l'immensité d'emblée insatiable et inassignable de l'être de l'homme, être qui est tout de désir noué comme s'il n'était que de la seule promesse d'une altérité réelle, la figure d'une quelconque «nature», que celle-ci lui soit donnée par la détermination d'un attribut dit "essentiel» choisi et privilégié parmi une indéfinité qui demeure toujours possible et ouverte, ou bien par l'attribution d'un ensemble de propriétés qui le constituerait dans les termes de ce que nous appelons un "propre» «(p. 79-80). La tragédie serait donc, «l'espace particulier où serait tenu en échec le concept du sujet» (p. 82).

Concepts. Mais pour lire dans la tragédie la mise en (à la) question du sujet, certains concepts sont indispensables, ne serait-ce que celui du sujet avant sa constitution. Si l'on joue des deux acceptions du verbe «être»: d'une part l'assertion d'identité ou d'égalité entre un sujet et un prédicat, d'autre part la déclaration d'être ou d'exister, le sujet constitué aura une figure permettant de le reconnaître et de le rendre identique aux prédicats qu'il est. Mais avant cette identification, le sujet n'est rien. Il ne sera qu'en se laissant investir par une sphère prédicative. Or «tout se passerait, dans le tragique sophocléen, comme s'il était impossible, la tragédie étant la monstration de cette impossibilité, pour un être, de se rendre égal, au point d'en réclamer la paternité, la causalité, la responsabilité, etc., bref, d'assumer proprement les connotations principales de notre concept de sujet, à ce dans quoi il s'est investi -à quoi de toute façon il appartient» (p. 95). Cette impossibilité est articulée par une logique qui joue de trois aspects: «a) l'être à; b) ce à quoi l'être à est, peut, doit ou croit être; enfin c) ce qui rend possible le rapport de l'un à l'autre et que nous appellerons: la dimension d'origine» (p. 102). Il faudra donc d'abord que ce dans quoi le «sujet» décide ou choisit de s'investir lui soit extérieur; puis que le «sujet" soit totalement ouvert à cete extériorité au point de pouvoir s'y perdre; et enfin que ce soit toujours depuis cette extériorité que le «sujet» consente à jouer son être.

Répétition. Le jeu de cette logique, et son enjeu, peuvent en effet être répétés, mais cette fois dans l'analyse de deux pièces: Ajax et Les Trachiniennes. Le premier cas permet de revenir sur la question de la manifestation du divin et de montrer que celui-ci n'est pas plus «sujet» que l'humain. Quant au second, il permet de placer le désir en position de véritable «sujet» tragique. 
Conclusion. Le sujet est inconstituable. La tragédie proteste «contre cet artefact (le sujet) qui est rigoureusement nécessaire à l'instauration de cette forme du politique connue sous le nom d'État» (p. 169). Sans rien opposer à l'ordre, elle montre le type de cruauté impliqué par la volonté d'un ordre. « La démocratie s'instaurera de la définition du citoyen de la société civile; la tragédie proliférera au temps de cette instauration. Elle sera terminée lorsque lui aura succédé une nouvelle rationalité à prétention d'universalité. L'entredeux persiste toujours, la tragédie demeure fascinante puisque au moment d'entrer sur la scène du monde, nous procédons tous comme Descartes. Larvatus prodeo. (p. 174-175).

Ce livre est beau. Matériellement d'abord, par sa facture particulièrement soignée. Mais surtout par son contenu. Cet effort d'écouter la tragédie comme elle a dû/pu se dire et s'entendre, cette tentative de "lire le sens du tragique au niveau de l'assertion d'identité et de la constitution de quelque chose comme un sujet» (p. 91), montre que si le troisième type d'interprétation relevé par Gravel est "le plus dangereux», il est aussi incontournable: on ne peut tramer le sens d'une question sans ourdir du même coup la question du sens. Un incontournable qui prend en conséquence l'allure d'un défi. Celui-ci, Gravel l'a relevé en produisant quelque chose comme un fragment de l'épistémè d'une époque dite révolue mais qui, loin de se réduire à quelque momie d'un quelconque musée du savoir, interpelle notre épistémè et sa/ses philosophies. $\mathrm{Ne}$ serait-ce que pour cette radicalité, et pour un plaisir d'écriture qui transparaît de façon peu banale même dans un lieu aussi inhabituel que l'index des «sujets», ce livre doit être lu.

Faculté de Philosophie

Université Laval 